\title{
EXTRAÇÃO E REFINO DE ÓLEO DE PESCADO OBTIDO A PARTIR DE RESÍDUOS DO BENEFICIAMENTO DE BAGRE (Ictalarus puntatus)
}

\author{
I.D. Agnol ${ }^{1}$, A.V. Igansi ${ }^{1}$, J.I. Engelmann ${ }^{1}$,L. A. A. Pinto ${ }^{1}$, T. R. S. Cadaval Jr. ${ }^{1}$ \\ ${ }^{1}$ Escola de Química e Alimentos, Universidade Federal do Rio Grande. \\ E-mail para contato: Igordallagnoleq@gmail.com
}

RESUMO - Este trabalho teve como objetivo extrair óleo bruto de cabeças de bagre (Ictalarus puntatus) através do processo termomecânico de produção de farinha de pescado, realizar o refino químico do óleo obtido e avaliar a qualidade dos óleos bruto e branqueado, através das análises químicas de acidez livre (AGL), índices de peróxido (IP), de iodo (II) e de saponificação (IS). As cabeças apresentaram teores $(\% \mathrm{~m} / \mathrm{m})$ de umidade de $69,90 \%$, proteína de $8,39 \%$, lipídios de $11,93 \%$ e cinzas de 9,78\%. O óleo bruto de cabeças apresentou AGL de 6,62\% em ácido oléico, IP de 13,08 meq/kg, IS de 189 $\mathrm{mg}_{\mathrm{KOH}} / \mathrm{g}$ e II de $126 \mathrm{cg}_{\mathrm{I} 2} / \mathrm{g}$, respectivamente. Já o óleo branqueado apresentou AGL de 0,23\%, IP 2,16 meq/kg, IS de $188 \quad \mathrm{mg}_{\mathrm{KOH}} / \mathrm{g}$ e II $127 \quad \mathrm{cg}_{\mathrm{I} 2} / \mathrm{g}$, respectivamente.Verificou-se que o conteúdo de AGL e IP foram reduzidos de maneira significativa $(\mathrm{p}<0,05)$, indicando que o refino foi eficiente em relação à remoção dos compostos que interferem negativamente na estabilidade físicoquímica do óleo de pescado.

\section{INTRODUÇÃO}

A busca por hábitos mais saudáveis tem impulsionado o uso de alimentos que auxiliam em uma melhor qualidade de vida. Em vista disso, o consumo de pescado apresenta uma valiosa fonte de proteínas e nutrientes, sendo considerado um produto de grande potencial para o consumo alimentar. Assim, a produção mundial de pescado mais que duplicou desde 1950 e, consequentemente, elevou a quantidade de resíduos gerados pelas indústrias de beneficiamento (FAO, 2016).

Os óleos de pescados apresentam diferentes perfis de ácidos graxos, os quais são geralmente determinados pelos tipos de ácidos graxos presentes na dieta destes pescados. Estes óleos são valorizados do ponto de vista nutricional pelo fato de possuírem conteúdos consideráveis de ácidos graxos poli-insaturados da família $\omega-3$ (EFSA, 2010; Averina e Kutyrev, 2011). Entretanto, o óleo bruto apresenta em sua composição impurezas, as quais interferem negativamente nas suas características físico-químicas e nutricionais, fazendo-se necessário submetê-lo ao processo de refino (degomagem, neutralização, lavagem, branqueamento e desodorização) a fim de que o produto obtido seja adequado para o 


\section{Congresso Brasileiro de Engenharia Química \\ em Iniciação Científica \\ UFSCar - São Carlos - SP

consumo humano (Crexi et al., 2010).

Neste contexto, este trabalho teve como objetivo extrair o óleo bruto de cabeças de bagre através de processo termomecânico, realizar o refino químico do óleo obtido e avaliar as características dos óleos bruto, degomado,neutralizado e branqueado, através das análises químicas de acidez livre (AGL), índices de peróxido (IP), de iodo (II) e de saponificação (IS).

\section{MATERIAL E METODOS}

\subsection{Material}

Os resíduos de pescado (cabeças) da espécie bagre (Ictalarus puntatus) foram obtidos por pescadores locais da cidade de Rio Grande/RS. Estes resíduos foram imediatamente refrigerados e transportados em recipiente adequado até o Laboratório de Tecnologia Industrial/FURG/RS, sendo armazenados sob congelamento até o momento do seu uso.

\subsection{Extração do óleo de pescado}

O óleo bruto de pescado foi obtido a partir do processo termomecânico, com condições de processos segundo Crexi et al. (2010). As cabeças foram descongeladas sob temperatura de refrigeração durante um período de $12 \mathrm{~h}$, após foram submetidas à cocção em autoclave durante $30 \mathrm{~min}$ sob temperaturas entre 95 e $100^{\circ} \mathrm{C}$, e em seguida as amostras foram prensadas para separação do licor de prensa dos sólidos. O licor de prensa foi centrifugado a 7000×g durante 20 min, obtendo-se ao final o óleo bruto de pescado.

\subsection{Refino do óleo de pescado}

O óleo bruto de cabeças de bagre obtido foi refinado de acordo com a metodologia descrita por Crexi et al. (2010), sendo composto pelas etapas de degomagem, neutralização, lavagem, secagem e branqueamento.

A etapa de degomagem ocorreu sob temperatura de $80^{\circ} \mathrm{C}, 500 \mathrm{rpm}$ e adição de $1 \%$ de ácido fosfórico $(85 \% \mathrm{p} / \mathrm{v})$ em relação a massa de óleo durante um período de $30 \mathrm{~min}$. As gomas foram separadas por centrifugação a $7000 \times \mathrm{g}$. A neutralização foi realizada sob temperatura de $40^{\circ} \mathrm{C}$, agitação de $500 \mathrm{rpm}$ e adição da solução aquosa de $\mathrm{NaOH} 20 \% \mathrm{~m} / \mathrm{m}$ (quantidade estequiométrica necessária para neutralizar os ácidos graxos livres presentes no óleo mais a quantidade de excesso em relação à acidez livre), mantendo-se tais condições durante um período de $20 \mathrm{~min}$. A fim de facilitar a separação da borra, elevou-se a temperatura do óleo até $80^{\circ} \mathrm{C}$ com posterior centrifugação a $7000 \times \mathrm{g}$ durante $20 \mathrm{~min}$. Na etapa de lavagem adicionou-se $10 \%$ de água $\left(95^{\circ} \mathrm{C}\right)$ em relação à massa de óleo, com agitação de $500 \mathrm{rpm}$, temperatura de $50^{\circ} \mathrm{C}$ mantendo-se um tempo de contato de $10 \mathrm{~min}$. Esta etapa foi realizada três vezes. Na etapa de secagem, o óleo neutralizado foi aquecido até $90{ }^{\circ} \mathrm{C}$ e agitação de $100 \mathrm{rpm}$, até completa evaporação da água (aproximadamente 10 min). A etapa de branqueamento subdividiu-se em duas etapas. Na primeira etapa, foi realizada a clarificação do óleo, sob temperatura de $70^{\circ} \mathrm{C}, 40 \mathrm{rpm}$ e adição de $5 \%$ de 
adsorvente (mistura de terra ativada e carvão ativado na relação de 9:1), durante um tempo de contato de 20 min. Após, foi feita a filtração do óleo em funil de Buchnner com uma pré-capa de terra diatomácea. Todas as etapas do processo de refino foram realizadas sob $700 \mathrm{~mm} \mathrm{Hg}$ de vácuo.

\subsection{Análises químicas}

A matéria-prima (cabeças de bagre) foram caracterizadas em relação a umidade (método 925.10), cinzas (método 945.46), proteínas (960.52), de acordo com a metodologia da AOAC de 1995. Para a determinação do teor de lipídios foi utilizado o método de BligheDyer(1959).

Os óleos bruto e branqueado das cabeças foram caracterizados em relação ao conteúdo de ácidos graxos livres em ácido oléico (AGL) (método Ca 5a - 40), índice de iodo (II) (método Cd 1-25), índice de saponificação (IS) (método Cd 36-76) e índice de peróxido (Cd 8-53), conforme as metodologias da AOCS (1980). Os resultados obtidos a partir da caracterização dos óleos brutos e branqueados de cabeças foram comparados usando o teste de Tukey de diferença de médias, utilizando o software Statistica 7.0 (StatSoft, EUA). Os valores foram considerados significativos a um nível de $95 \%$ ( $p$ < $0,05)$.

\section{RESULTADOS E DISCUSSÃO}

A composição centesimal das cabeças de bagrepode ser observada na Tabela 1.

Tabela 1 - Composição centesimal das cabeças de bagre.

\begin{tabular}{|c|c|}
\hline Composição & Valores obtidos \\
\hline \hline Umidade (\%) & $69,9 \pm 1,5$ \\
\hline Cinzas (\%) & $9,8 \pm 0,9$ \\
\hline Proteínas (\%) & $8,4 \pm 1,3$ \\
\hline Lipídios (\%) & $11,9 \pm 0,7$ \\
\hline
\end{tabular}

Valor médio \pm desvio padrão.

Os valores encontrados na Tabela 1 podem sofrer variações, que estão diretamente relacionadas com a parte do corpo do pescado. Sendo verificado que as cabeças apresentam maior quantidade de ossos, acarretando em uma maior quantidade de cinzas, e menor quantidade de proteínas e umidade, quando comparados com as vísceras de bagre (Reidelet al.,2010). Além disso, estas características podem sofrer alterações relacionadas com os locais de desenvolvimento do pescado, condições ambientais, idade, sexo, influenciando diretamente na composição química do mesmo, principalmente no que tange a fração lipídica (Druzian et al., 2007).

Na Tabela 2 podem ser observados os resultados obtidos a partir da caracterização dos óleos brutos e branqueados de cabeças de bagre. 
Tabela 2 - Resultados obtidos a partir da caracterização química do óleo de bagre ao longo do processo refino

\begin{tabular}{|c|c|c|c|c|}
\hline & $\begin{array}{c}\text { Óleo } \\
\text { Bruto }\end{array}$ & ÓleoDegomado & $\begin{array}{c}\text { Óleo } \\
\text { Neutralizado }\end{array}$ & $\begin{array}{c}\text { Óleo } \\
\text { Branqueado }\end{array}$ \\
\hline \hline $\mathrm{AGL}\left(\%_{\text {acidooléico }}\right)$ & $6,62 \pm 0,04^{\mathrm{a}}$ & $6,41 \pm 0,24^{\mathrm{a}}$ & $0,24 \pm 0,01^{\mathrm{b}}$ & $0,23 \pm 0,01^{\mathrm{b}}$ \\
\hline $\mathrm{II}(\mathrm{cg}) \mathrm{I}_{2} / \mathrm{g}$ & $126 \pm 3^{\mathrm{a}}$ & $126 \pm 2^{\mathrm{a}}$ & $127 \pm 1^{\mathrm{a}}$ & $127 \pm 2^{\mathrm{a}}$ \\
\hline $\mathrm{IS}\left(\mathrm{mg}_{\mathrm{KOH}} / \mathrm{g}\right)$ & $189 \pm 2^{\mathrm{a}}$ & $188 \pm 1^{\mathrm{a}}$ & $189 \pm 2^{\mathrm{a}}$ & $189 \pm 1^{\mathrm{a}}$ \\
\hline $\mathrm{IP}\left(\mathrm{m}_{\mathrm{eq}} / \mathrm{kg}\right)$ & $13,18 \pm 0,01^{\mathrm{a}}$ & $12,52 \pm 0,14^{\mathrm{b}}$ & $7,86 \pm 0,03^{\mathrm{c}}$ & $2,16 \pm 0,01^{\mathrm{d}}$ \\
\hline
\end{tabular}

Valor médio \pm desvio padrão. Letras diferentes na mesma linha apresentam diferenças significativas $(\mathrm{p}<0,05)$.

Na Figura 1 podem ser observados os óleos bruto e branqueado de cabeças de bagre, a temperatura de $25^{\circ} \mathrm{C}$, respectivamente.

Figura 1 - (a) Óleo bruto de cabeças de bagre, (b) Óleo branqueado de cabeças de bagre

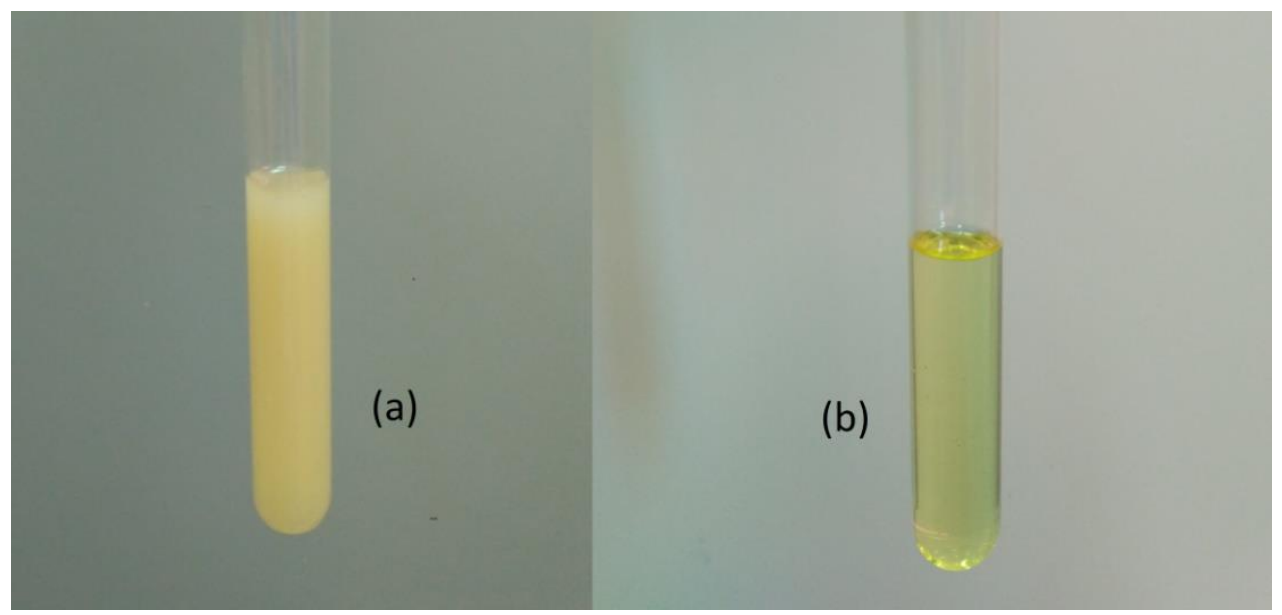

A partir da Tabela 2, pode-se observar que o óleo bruto apresentou AGL mais elevado, quando comparados aos óleos neutralizado e branqueado. Em relação ao conteúdo de AGL do óleo branqueado, pode-se verificar que o processo de refino reduziu os níveis destes de forma significativa $(p<0,05)$, e abaixo do máximo permitido pela legislação $(0,3 \%$ em ácido oléico) (ANVISA, 2005). Esta redução pode ser explicada pelo fato da etapa de neutralização ter como objetivo a remoção dos AGL presentes no óleo. Além disso, a etapa de branqueamento funciona como um polimento, removendo a borra residual proveniente da neutralização (Rossi et al., 2003).O II representa o grau de insaturação do óleo, enquanto que o IS indica a massa molar média dos ácidos graxos esterificados ao glicerol (AOCS, 1980). No que diz respeito a estes índices pode-se observar que não houve variações significativas $(\mathrm{p}>0,05)$ ao longo do processo de refino, visto que as etapas que compõe este processo não alteram a composição de ácidos graxos dos triacilgliceróis. 
O IP indica o estágio inicial da oxidação lipídica, sendo um importante parâmetro por definir o estado da deterioração de óleos e gorduras (Manral et al., 2008). Na Tabela 2 pode-se observar que este índice foi reduzido significativamente ao longo do processo de refino do óleo, estando abaixo dos níveis especificados pela legislação que é de 10 $\mathrm{meq}_{\text {peroxido}} / \mathrm{kgóleo.} \mathrm{A} \mathrm{redução} \mathrm{dos} \mathrm{níveis} \mathrm{de} \mathrm{peróxido} \mathrm{deve-se} \mathrm{principalmente} \mathrm{a} \mathrm{etapa} \mathrm{de}$ branqueamento do óleo, na qual são utilizados adsorventes que auxiliam na remoção de compostos de oxidação, como os peróxidos e cetonas (Pestana-Bauer, 2012). Além da remoção dos compostos de oxidação, pode-se observar que o branqueamento foi eficiente em relação à remoção dos compostos que conferem coloração escura ao óleo, conforme pode ser visualizado nas Figuras 1 (b).

\section{CONCLUSÃO}

As cabeças de bagre utilizadas para a realização deste trabalho apresentaram 69,9\% de umidade, $9,8 \%$ de cinzas, $8,4 \%$ de proteína e 11,9\% de lipídios. Através das análises químicas foi verificado que o percentual AGL e de peróxidos do óleo bruto foram reduzidos significativamente $(\mathrm{p}<0,05)$ após o processo de refino do óleo, apresentando resultados inferiores aos limites máximos exigidos pela legislação. O II e IS não variaram após o refino, o que já era esperado, visto que este processo não altera a composição de ácidos graxos do triacilglicerol. Com base nestes resultados pode-se afirmar que refino realizado melhorou as características dos óleos obtidos, visto que removeu componentes como ácidos graxos livres e produtos de oxidação lipídica, tornando o óleo refinado de acordo com as especificações para consumo, conforme a legislação. Portanto, conclui-se que a obtenção de óleo de pescado através de cabeças de bagre representa uma boa alternativa para o aproveitamento dos resíduos gerados durante o beneficiamento destes pescados.

\section{REFERENCIAS BIBLIOGRÁFICAS}

ANVISA - Agência Nacional de Vigilância Sanitária, Resolução nº 270, de 22 de setembro de 2005 estabelece o regulamento técnico para óleos vegetais, gorduras vegetais e creme vegetal. Diário Oficial da União, Poder Executivo, Publicada 23 de setembro de 2005.

AOAC. Association of Official Analytical Chemists. Official methods of analysis of A.O.A.C. International. 16 ed. Arlington, 1995.

AOAC. Association of Official Analytical Chemists. Official methods of analysis of A.O.A.C. International. 16 ed. Arlington, 1980.

AOCS. American Oil Chemists Society. Official and Tentative Methods of American Oil Chem. Soc. 5 ed. Champaign, 2002. 


\section{Congresso Brasileiro de Engenharia Química em Iniciação Científica UFSCar - São Carlos - SP 16 a 19 de Julho de 2017}

AVERINA, E. S.; KUTYREV, I. A. Perspectives of using of marine and freshwater hydrobionts oils for development of drug delivery systems. Biotechnol. Adv., 2011.

CREXI V.T.; MONTE, M. L.; SOUZA-SOARES, L. A.; PINTO, L.A.A. Production and refinement of oil from carp (Cyprinuscarpio) viscera. FoodChem., v. 119, p. 945-950, 2010.

DRUZIAN, J. I.; MARCHESI, C. M.; SCAMPARINI, A. R. P. Perfil de ácidos graxos e composição centesimal de carpas (Cyprinuscarpio) alimentadas com ração e com dejetos suínos. Cienc. Rural, v.37, n.2, p.539-544, 2007.

EUROPEAN FOOD SAFETY AUTHORITY (EFSA). Scientific opinion on Fish Oil for Human Consumption. Food Hygiene, including Rancidity. EFSA J., v. 8, n. 10, p. 1874-1922, 2010.

FAO. Food and Agriculture Organization of the United Nations.Fishandseafoodutilization,2016. Disponível em: http://www.fao.org/fishery/utilization/en acesso em 29/03/2017.

MANRAL, M.; PANDEY, M. C.; JAYATHILAKAN, K.; RADHAKRISHNA, K.; BAWA, A. S. Effect of fish (Catlacatla) fring on the quality characteristics of sunflower oil. Food Chem., v. 106, p. 634-639, 2008.

PESTANA-BAUER, V. R.; ZAMBIAZI, R. C.; MENDONÇA, C. R. B.; BENEITOCAMBRA, M.; RAMIS-RAMOS, G. $\gamma$-oryzanol and tocopherol contents in residues of rice bran oil refining. FoodChem.,v. 134, p. 1479-1483, 2012.

REIDEL, A.; ROMAGOSA, E.; FEIDEN, A.; BOSCOLO, W. R.; COLDEBELLA, A.; SIGNOR, A.A. Rendimento corporal e composição química de jundiás alimentados com diferentes níveis de proteína e energia na dieta, criados em tanques-rede. Rev.Bras. Zootec., v.39, n. 2, p.233-240, 2010.

ROSSI, M.; GIANAZZA, M.; ALAMPRESE, C.; STANGA, F. The role of bleaching clays and synthetic sílica in palm oil physical refining. Food Chem., v. 82, p. 291-293, 2003. 